\title{
Supersymmetric Quintessence
}

\author{
Francesca Rosati* \\ SISSA, via Beirut 2-4, I-34013 Trieste, ITALY \\ INFN, sezione di Trieste, Padriciano 99, I-34014 Trieste, ITALY \\ E-mail: rosati@sissa.it
}

Abstract: Recent data point in the direction of a cosmological constant dominated universe. We investigate the rôle of supersymmetric QCD with $N_{f}<N_{c}$ as a possible candidate for dynamical cosmological constant ("quintessence"). We take in full consideration the multi-scalar nature of the model, allowing for different initial conditions for the $N_{f}$ independent scalar VEVs and studying the coupled system of $N_{f}$ equations of motion. The issues related to the coupling of the scalars with other cosmological fields are also addressed.

\section{Introduction}

Indications for an accelerating universe coming from redshift-distance measurements of High-Z Supernovae Ia (SNe Ia) [2, 3], combined with CMB data [4] and cluster mass distribution [5], have recently drawn a great deal of attention on cosmological models with $\Omega_{m} \sim 1 / 3$ and $\Omega_{\Lambda} \sim$ $2 / 3, \Omega_{m}$ and $\Omega_{\Lambda}$ being the fraction densities in matter and cosmological constant, respectively. More generally, the rôle of the cosmological constant in accelerating the universe expansion could be played by any smooth component with negative equation of state $p_{Q} / \rho_{Q}=w_{Q} \lesssim-0.6$ $[6,7]$, as in the so-called "quintessence" models (QCDM) [7], otherwise known as $x \mathrm{CDM}$ models [9].

A natural candidate for quintessence is given by a rolling scalar field $Q$ with potential $V(Q)$ and equation of state

$$
w_{Q}=\frac{\dot{Q}^{2} / 2-V(Q)}{\dot{Q}^{2} / 2+V(Q)}
$$

which - depending on the amount of kinetic energy - could in principle take any value from -1 to +1 . The study of scalar field cosmologies has shown $[10,11]$ that for certain potentials there exist attractor solutions that can be of the "scaling" $[12,13,14]$ or "tracker" $[15,16]$ type; that

* Report on work done in collaboration with Antonio Masiero and Massimo Pietroni [1]. means that for a wide range of initial conditions the scalar field will rapidly join a well defined late time behavior.

If $\rho_{Q} \ll \rho_{B}$, where $\rho_{B}$ is the energy density of the dominant background (radiation or matter), the attractor can be studied analytically.

In the case of an exponential potential, $V \sim$ $\exp (-Q)$ the solution $Q \sim \ln t$ is, under very general conditions, a "scaling" attractor in phase space characterized by $\rho_{Q} / \rho_{B} \sim$ const $[12,13$, 14]. This could potentially solve the so called "cosmic coincidence" problem, providing a dynamical explanation for the order of magnitude equality between matter and scalar field energy today. Unfortunately, the equation of state for this attractor is $w_{Q}=w_{B}$, which cannot explain the acceleration of the universe neither during $\mathrm{RD}\left(w_{\text {rad }}=1 / 3\right)$ nor during $\mathrm{MD}\left(w_{m}=0\right)$. Moreover, Big Bang nucleosynthesis constrain the field energy density to values much smaller than the required $\sim 2 / 3[11,13,14]$.

If instead an inverse power-law potential is considered, $V=M^{4+\alpha} Q^{-\alpha}$, with $\alpha>0$, the attractor solution is $Q \sim t^{1-n / m}$, where

$$
n=3\left(w_{Q}+1\right), \quad m=3\left(w_{B}+1\right) ;
$$

and the equation of state turns out to be

$$
w_{Q}=\frac{w_{B} \alpha-2}{\alpha+2}
$$


which is always negative during MD. The ratio of the energies is no longer constant but scales as $\rho_{Q} / \rho_{B} \sim a^{m-n}$ thus growing during the cosmological evolution, since $n<m$. $\rho_{Q}$ could then have been safely small during nucleosynthesis and have grown lately up to the phenomenologically interesting values. These solutions are then good candidates for quintessence and have been denominated "tracker" in the literature $[11,15,16]$.

The inverse power-law potential does not improve the cosmic coincidence problem with respect to the cosmological constant case. Indeed, the scale $M$ has to be fixed from the requirement that the scalar energy density today is exactly what is needed. This corresponds to choosing the desired tracker path. An important difference exists in this case though. The initial conditions for the physical variable $\rho_{Q}$ can vary between the present critical energy density $\rho_{c r}^{0}$ and the background energy density $\rho_{B}$ at the time of beginning [16] (this range can span many tens of orders of magnitude, depending on the initial time), and will anyway end on the tracker path before the present epoch, due to the presence of an attractor in phase space $[15,16]$. On the contrary, in the cosmological constant case, the physical variable $\rho_{\Lambda}$ is fixed once for all at the beginning. This allows us to say that in the quintessence case the fine-tuning issue, even if still far from solved, is at least weakened.

A great effort has recently been devoted to find ways to constrain such models with present and future cosmological data in order to distinguish quintessence from $\Lambda$ models $[17,18]$. An even more ambitious goal is the partial reconstruction of the scalar field potential from measuring the variation of the equation of state with increasing redshift [19].

On the other hand, the investigation of quintessence models from the particle physics point of view is just in a preliminary stage and a realistic model is still missing (see for example refs. $[20,21,22,23])$. There are two classes of problems: the construction of a field theory model with the required scalar potential and the interaction of the quintessence field with the standard model (SM) fields [24]. The former problem was already considered by Binétruy [20], who pointed out that scalar inverse power law po- tentials appear in supersymmetric QCD theories (SQCD) [25] with $N_{c}$ colors and $N_{f}<N_{c}$ flavors. The latter seems the toughest. Indeed the quintessence field today has typically a mass of order $H_{0} \sim 10^{-33} \mathrm{eV}$. Then, in general, it would mediate long range interactions of gravitational strength, which are phenomenologically unacceptable.

In this talk, both theese issue will be addressed, following the results obtained in ref. [1].

\section{SUSY QCD}

As already noted by Binètruy [20], supersymmetric QCD theories with $N_{c}$ colors and $N_{f}<N_{c}$ flavors [25] may give an explicit realization of a model for quintessence with an inverse power law scalar potential. The remarkable feature of these theories is that the superpotential is exactly known non-perturbatively. Moreover, in the range of field values that will be relevant for our purposes (see below) quantum corrections to the Kähler potential are under control. As a consequence, we can study the scalar potential and the field equations of motion of the full quantum theory, without limiting ourselves to the classical approximation.

The matter content of the theory is given by the chiral superfields $Q_{i}$ and $\bar{Q}_{i}\left(i=1 \ldots N_{f}\right)$ transforming according to the $N_{c}$ and $\bar{N}_{c}$ representations of $S U\left(N_{c}\right)$, respectively. In the following, the same symbols will be used for the superfields $Q_{i}, \bar{Q}_{i}$, and their scalar components.

Supersymmetry and anomaly-free global symmetries constrain the superpotential to the unique exact form

$$
W=\left(N_{c}-N_{f}\right)\left(\frac{\Lambda^{\left(3 N_{c}-N_{f}\right)}}{\operatorname{det} T}\right)^{\frac{1}{N_{c}-N_{f}}}
$$

where the gauge-invariant matrix superfield $T_{i j}=$ $Q_{i} \cdot \bar{Q}_{j}$ appears. $\Lambda$ is the only mass scale of the theory. It is the supersymmetric analogue of $\Lambda_{Q C D}$, the renormalization group invariant scale at which the gauge coupling of $S U\left(N_{c}\right)$ becomes non-perturbative. As long as scalar field values $Q_{i}, \bar{Q}_{i} \gg \Lambda$ are considered, the theory is in the weak coupling regime and the canonical form for the Kähler potential may be assumed. The scalar 
and fermion matter fields have then canonical kinetic terms, and the scalar potential is given by

$$
V=\sum_{i=1}^{N_{f}}\left(\left|F_{Q_{i}}\right|^{2}+\left|F_{\bar{Q}_{i}}\right|^{2}\right)+\frac{1}{2} D^{a} D^{a}
$$

where $F_{Q_{i}}=\partial W / \partial Q_{i}, F_{\bar{Q}_{i}}=\partial W / \partial \bar{Q}_{i}$, and

$$
D^{a}=Q_{i}^{\dagger} t^{a} Q_{i}-\bar{Q}_{i} t^{a} \bar{Q}_{i}^{\dagger} .
$$

The relevant dynamics of the field expectation values takes place along directions in field space in which the above D-term vanish, i.e. the perturbatively flat directions $\left\langle Q_{i \alpha}\right\rangle=\left\langle\bar{Q}_{i \alpha}^{\dagger}\right\rangle$, where $\alpha=1 \cdots N_{c}$ is the gauge index. At the nonperturbative level these directions get a non vanishing potential from the F-terms in (2.2), which are zero at any order in perturbation theory.

Gauge and flavor rotations can be used to diagonalize the $\left\langle Q_{i \alpha}\right\rangle$ and put them in the form

$$
\left\langle Q_{i \alpha}\right\rangle=\left\langle\bar{Q}_{i \alpha}^{\dagger}\right\rangle=\begin{array}{ll}
q_{i} \delta_{i \alpha} & 1 \leq \alpha \leq N_{f} \\
0 & N_{f} \leq \alpha \leq N_{c}
\end{array} .
$$

Along these directions, the scalar potential is given by

$v\left(q_{i}\right) \equiv\left\langle V\left(Q_{i}, \bar{Q}_{i}\right)\right\rangle=\frac{2 \Lambda^{2 a}}{\prod_{i=1}^{N_{f}}\left|q_{i}\right|^{4 d}}\left(\sum_{j=1}^{N_{f}} \frac{1}{\left|q_{j}\right|^{2}}\right)$,

with

$$
a=\frac{3 N_{c}-N_{f}}{N_{c}-N_{f}}, \quad d=\frac{1}{N_{c}-N_{f}} .
$$

In the following, we will be interested in the cosmological evolution of the $N_{f}$ expectation values $q_{i}$, given by

$$
\left\langle\ddot{Q}_{i}+3 H \dot{Q}_{i}+\frac{\partial V}{\partial Q_{i}^{\dagger}}\right\rangle=0, i=1, \ldots, N_{f} .
$$

In Ref. [20] the same initial conditions for all the $N_{f}$ VEV's and their time derivatives were chosen. With this very peculiar choice the evolution of the system may be described by a single VEV $q$ (which we take real) with equation of motion

$$
\ddot{q}+3 H \dot{q}-g \frac{\Lambda^{2 a}}{q^{2 g+1}}=0, \quad g=\frac{N_{c}+N_{f}}{N_{c}-N_{f}},
$$

thus reproducing exactly the case of a single scalar field $\Phi$ in the potential $V=\Lambda^{4+2 g} \Phi^{-2 g} / 2$ considered in refs. $[10,11,16]$. We will instead consider the more general case in which different initial conditions are assigned to different VEV's, and the system is described by $N_{f}$ coupled differential equations. Taking for illustration the case $N_{f}=2$, we will have to solve the equations

$$
\begin{aligned}
& \ddot{q}_{1}+3 H \dot{q}_{1}-\frac{d \cdot q_{1} \Lambda^{2 a}}{\left(q_{1} q_{2}\right)^{2 d N_{c}}}\left[2+N_{c} \frac{q_{2}^{2}}{q_{1}^{2}}\right]=0, \\
& \ddot{q}_{2}+3 H \dot{q}_{2}-\frac{d \cdot q_{2} \Lambda^{2 a}}{\left(q_{1} q_{2}\right)^{2 d N_{c}}}\left[2+N_{c} \frac{q_{1}^{2}}{q_{2}^{2}}\right]=0(2.5)
\end{aligned}
$$

with $H^{2}=8 \pi / 3 M_{P}^{2}\left(\rho_{m}+\rho_{r}+\rho_{Q}\right)$, where $M_{P}$ is the Planck mass, $\rho_{m(r)}$ is the matter (radiation) energy density, and $\rho_{Q}=2\left(\dot{q}_{1}^{2}+\dot{q}_{2}^{2}\right)+v\left(q_{1}, q_{2}\right)$ is the total field energy.

\section{The tracker solution}

In analogy with the one-scalar case, we look for power-law solutions of the form

$$
q_{t r, i}=C_{i} \cdot t^{p_{i}}, \quad i=1, \cdots, N_{f} .
$$

It is straightforward to verify that - when $\rho_{Q} \ll$ $\rho_{B}$ - the only solution of this type is given, for $i=1, \cdots, N_{f}$, by

$p_{i} \equiv p=\frac{1-r}{2}, \quad C_{i} \equiv C=\left[X^{1-r} \Lambda^{2(3-r)}\right]^{1 / 4}$,

with

$$
X \equiv \frac{4 m(1+r)}{(1-r)^{2}[12-m(1+r)]},
$$

where we have defined

$$
r \equiv \frac{N_{f}}{N_{c}}\left(=\frac{1}{N_{c}}, \ldots, 1-\frac{1}{N_{c}}\right) .
$$

This solution is characterized by an equation of state

$$
w_{Q}=\frac{1+r}{2} w_{B}-\frac{1-r}{2} .
$$

Following the same methods employed in ref. [11] one can show that the above solution is the unique stable attractor in the space of solutions of eqs. (2.5). Then, even if the $q_{i}$ 's start with different initial conditions, there is a region in field configuration space such that the system evolves towards the equal fields solutions (3.1), and the late-time behavior is indistinguishable from the case considered in ref. [20]. 
The field energy density grows with respect to the matter energy density as

$$
\frac{\rho_{Q}}{\rho_{m}} \sim a^{\frac{3(1+r)}{2}},
$$

where $a$ is the scale factor of the universe. The scalar field energy will then eventually dominate and the approximations leading to the scaling solution (3.1) will drop, so that a numerical treatment of the field equations is mandatory in order to describe the phenomenologically relevant latetime behavior.

The scale $\Lambda$ can be fixed requiring that the scalar fields are starting to dominate the energy density of the universe today and that both have already reached the tracking behavior. The two conditions are realized if

$$
v\left(q_{0}\right) \simeq \rho_{\text {crit }}^{0}, \quad v^{\prime \prime}\left(q_{0}\right) \simeq H_{0}^{2},
$$

where $\rho_{\text {crit }}^{0}=3 M_{P}^{2} H_{0}^{2} / 8 \pi$ and $q_{0}$ are the present critical density and scalar fields VEV respectively. Eqs. (3.4) imply

$$
\begin{aligned}
& \frac{\Lambda}{M_{P}} \simeq\left[\frac{3(1+r)(3+r)}{4 \pi(1-r)^{2} r N_{c}}\right]^{\frac{1+r}{2(3-r)}}\left(\frac{1}{2 r N_{c}} \frac{\rho_{c r i t}^{0}}{M_{P}^{4}}\right)^{\frac{1-r}{2(3-r)}} \\
& \frac{q_{0}^{2}}{M_{P}^{2}} \simeq \frac{3}{4 \pi} \frac{(1+r)(3+r)}{(1-r)^{2}} \frac{1}{r N_{c}} .
\end{aligned}
$$

Depending on the values for $N_{f}$ and $N_{c}, \Lambda$ and $q_{0} / \Lambda$ assume widely different values. $\Lambda$ takes its lowest possible values in the $N_{c} \rightarrow \infty\left(N_{f}\right.$ fixed) limit, where it equals $4 \cdot 10^{-2}\left(h^{2} / N_{f}^{2}\right)^{1 / 6}$ $\mathrm{GeV}$ (we have used $\left.\rho_{\text {crit }}^{0} / M_{P}^{4}=\left(2.5 \cdot 10^{-31} h^{1 / 2}\right)^{4}\right)$. For fixed $N_{c}$, instead, $\Lambda$ increases as $N_{f}$ goes from 1 to its maximum allowed value, $N_{f}=$ $1-N_{c}$. For $N_{c} \gtrsim 20$ and $N_{f}$ close to $N_{c}$, the scale $\Lambda$ exceeds $M_{P}$.

The accuracy of the determination of $\Lambda$ given in (3.5) depends on the present error on the measurements of $H_{0}$, i.e., typically,

$$
\frac{\delta \Lambda}{\Lambda}=\frac{1-r}{3-r} \frac{\delta H_{0}}{H_{0}} \lesssim 0.1 .
$$

In deriving the scalar potential (2.2) and the field equations (2.5) we have assumed that the system is in the weakly coupled regime, so that the canonical form for the Kähler potential may be considered as a good approximation. This condition is satisfied as long as the fields' VEVs are much larger than the non-perturbative scale $\Lambda$. From eq. (3.5) one can compute the ratio between the VEVs today and $\Lambda$, and see that it is greater than unity for any $N_{f}$ as long as $N_{c} \lesssim 20$.

\section{Interaction with the visible sector}

The superfields $Q_{i}$ and $\bar{Q}_{i}$ have been taken as singlets under the SM gauge group. Therefore, they may interact with the visible sector only gravitationally, i.e. via non-renormalizable operators suppressed by inverse powers of the Planck mass, of the form

$$
\int d^{4} \theta K^{j}\left(\phi_{j}^{\dagger}, \phi_{j}\right) \cdot \beta^{j i}\left[\frac{Q_{i}^{\dagger} Q_{i}}{M_{P}^{2}}\right],
$$

where $\phi_{j}$ represents a generic standard model superfield. From (3.5) we know that today the VEV's $q_{i}$ are typically $O\left(M_{P}\right)$, so there is no reason to limit ourselves to the contributions of lowest order in $|Q|^{2} / M_{P}^{2}$. Rather, we have to consider the full (unknown) functions $\beta$ 's and the analogous $\bar{\beta}$ 's for the $\bar{Q}_{i}$ 's. Moreover, the requirement that the scalar fields are on the tracking solution today, eqs. (3.4), implies that their mass is of order $\sim H_{0}^{2} \sim 10^{-33} \mathrm{eV}$.

The exchange of very light fields gives rise to long-range forces which are constrained by tests on the equivalence principle, whereas the time dependence of the VEV's induces a time variation of the SM coupling constants [24, 27]. These kind of considerations set stringent bounds on the first derivatives of the $\beta^{j i}$ 's and $\bar{\beta}^{j i}$ 's today,

$$
\begin{gathered}
\left.\alpha^{j i} \equiv \frac{d \log \beta^{j i}\left[x_{i}^{2}\right]}{d x_{i}}\right|_{x_{i}=x_{i}^{0}}, \\
\left.\bar{\alpha}^{j i} \equiv \frac{d \log \bar{\beta}^{j i}\left[x_{i}^{2}\right]}{d x_{i}}\right|_{x_{i}=x_{i}^{0}},
\end{gathered}
$$

where $x_{i} \equiv q_{i} / M_{P}$. To give an example, the best bound on the time variation of the fine structure constant comes from the Oklo natural reactor. It implies that $|\dot{\alpha} / \alpha|<10^{-15} \mathrm{yr}^{-1}$ [28], leading to the following constraint on the coupling with the kinetic terms of the electromagnetic vector superfield $V$,

$$
\alpha^{V i}, \bar{\alpha}^{V i} \lesssim 10^{-6} \frac{H_{0}}{\left\langle\dot{q}_{i}\right\rangle} M_{P},
$$




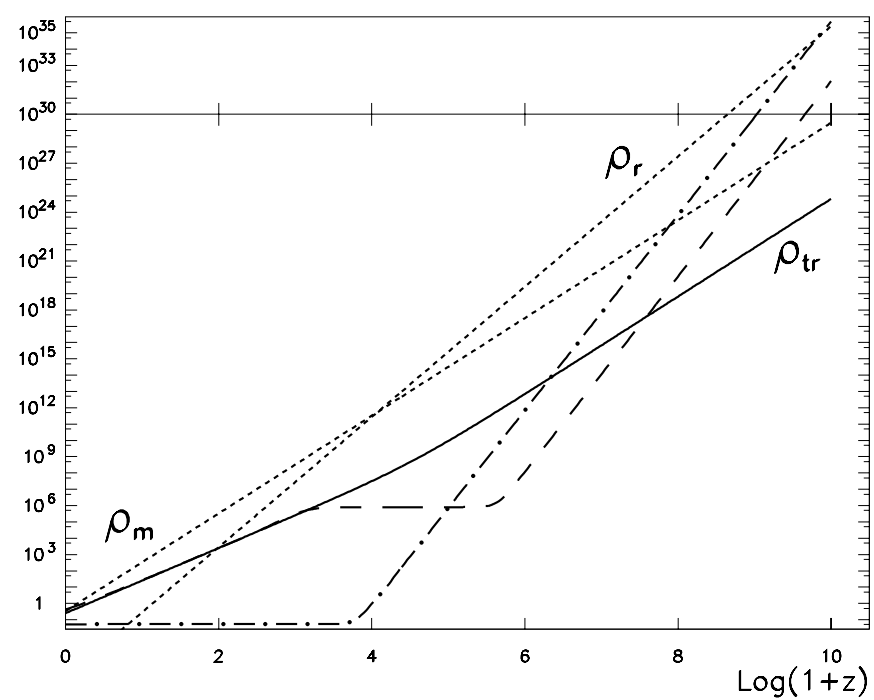

Figure 1: The evolution of the energy densities $\rho$ of different cosmological components is given as a funcion of red-shift. All the energy densities are normalized to the present critical energy density $\rho_{c r}^{0}$. Radiation and matter energy densities are represented by the short-dashed lines, whereas the solid line is the energy density of the tracker solution discussed in Section 3. The long-dashed line is the evolution of the scalar field energy density for a solution that reaches the tracker before the present epoch; while the dash-dotted line represents the evolution for a solution that overshoots the tracker to such an extent that it has not yet had enough time to re-join the attractor.

where $\left\langle\dot{q}_{i}\right\rangle$ is the average rate of change of $q_{i}$ in the past $2 \times 10^{9} \mathrm{yr}$.

Similar -although generally less stringentbounds can be analogously obtained for the coupling with the other standard model superfields [27]. Therefore, in order to be phenomenologically viable, any quintessence model should postulate that all the unknown couplings $\beta^{j i}$ 's and $\bar{\beta}^{j i}$ s s have a common minimum close to the actual value of the $q_{i}{ }^{\prime}{ }^{1}$.

The simplest way to realize this condition would be via the least coupling principle introduced by Damour and Polyakov for the massless superstring dilaton in ref. [26], where a universal coupling between the dilaton and the SM fields was postulated. In the present context, we will invoke a similar principle, by postulating that $\beta^{j i}=\beta$ and $\bar{\beta}^{j i}=\bar{\beta}$ for any SM field $\phi_{j}$ and any flavor $i$. For simplicity, we will further assume $\beta=\bar{\beta}$.

The decoupling from the visible sector implied by bounds like (4.2) does not necessarily

\footnotetext{
${ }^{1}$ An alternative way to suppress long-range interactions, based on an approximate global symmetry, was proposed in ref. [24].
}

mean that the interactions between the quintessence sector and the visible one have always been phenomenologically irrelevant. Indeed, during radiation domination the VEVs $q_{i}$ were typically $\ll M_{P}$ and then very far from the postulated minimum of the $\beta$ 's. For such values of the $q_{i}$ 's the $\beta$ 's can be approximated as

$$
\beta\left[\frac{Q^{\dagger} Q}{M_{P}^{2}}\right]=\beta_{0}+\beta_{1} \frac{Q^{\dagger} Q}{M_{P}^{2}}+\ldots
$$

where the constants $\beta_{0}$ and $\beta_{1}$ are not directly constrained by (4.2). The coupling between the (4.3) and the SM kinetic terms, as in (4.1), induces a SUSY breaking mass term for the scalars of the form [29]

$$
\Delta L \sim H^{2} \beta_{1} \sum_{i}\left(\left|Q_{i}\right|^{2}+\left|\bar{Q}_{i}\right|^{2}\right),
$$

where we have used the fact that during radiation domination $\left\langle\sum_{j} \int d^{4} \theta K^{j}\left(\phi_{j}^{\dagger}, \phi_{j}\right)\right\rangle \sim \rho_{\text {rad }}$.

If present, this term would have a very interesting impact on the cosmological evolution of the fields. First of all one should notice that, unlike the usual mass terms with time-independent masses considered in [22], a mass $m^{2} \sim H^{2}$ does 


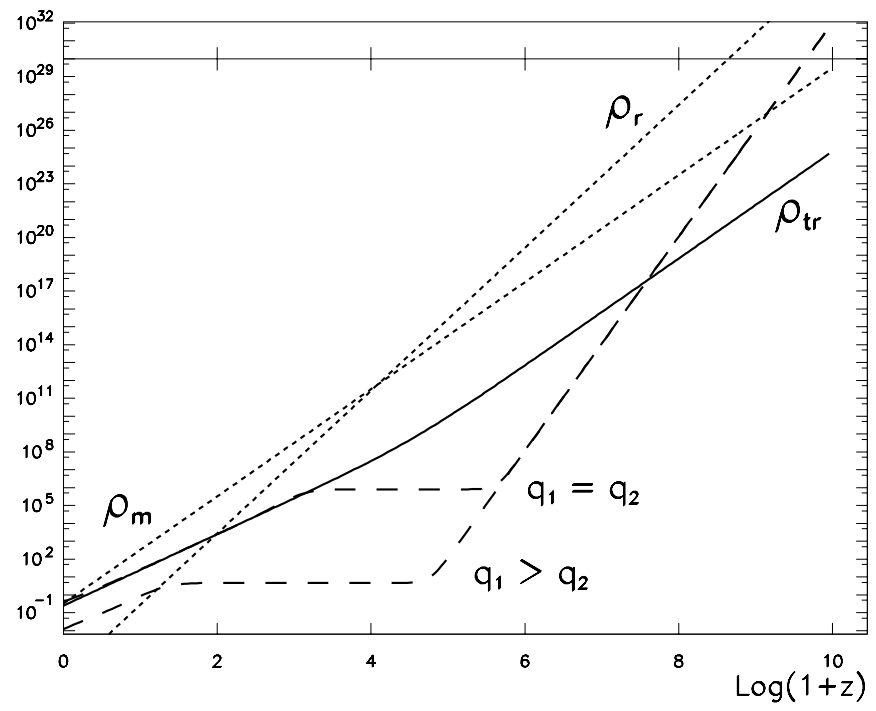

Figure 2: The effect of taking different initial conditions for the fields, at the same initial total field energy. Starting with $q_{1}^{i n} / q_{2}^{i n}=10^{15}$ the tracker behaviour is not reached today. For comparison we plot the solution for $q_{1}^{i n} / q_{2}^{i n}=1$.

not modify the time-dependence of the tracking solution, which is still the power-law given in eq. (3.1). Thus, the fine-tuning problems induced by the requirement that a soft-supersymmetry breaking mass does not spoil the tracking solutions [22] are not present here.

Secondly, since the $Q$ and $\bar{Q}$ fields do not form an isolated system any more, the equation of state of the quintessence fields is not linked to the power-law dependence of the tracking solution. Taking into account the interaction with the SM fields, represented by $H^{2}$, we find the new equation of state during radiation domination $\left(w_{B}=1 / 3\right)$

$$
w_{Q}^{\prime}=w_{Q}-4 \beta_{1} \frac{1+r}{9(1-r)+6 \beta_{1}}
$$

where $w_{Q}$ was given in eq. (3.2).

From a phenomenological point of view, the most relevant effect of the presence of mass terms like (4.4) during radiation domination resides in the fact that they rise the scalar potential at large fields values, inducing a (time-dependent) minimum. In absence of such terms, if the fields are initially very far from the origin, they are not able to catch up with the tracking behavior before the present epoch, and $\rho_{Q}$ always remains much smaller than $\rho_{B}$. These are the wellknown 'undershoot' solutions considered in ref.
[16]. Instead, when large enough masses (4.4) are present, the fields are quickly driven towards the time-dependent minimum and the would-be undershoot solutions reach the tracking behavior in time.

The same happens for the would-be 'overshoot' solutions, [16], in which the fields are initially very close to the origin, with an energy density much larger than the tracker one, and are subsequently pushed to very large values, from where they will not be able to reach the tracking solution before the present epoch. Introducing mass terms like (4.4) prevents the fields to go to very large values, and keeps them closer to the traking solution.

In other words, the already large region in initial condition phase space leading to late-time tracking behavior, will be enlarged to the full phase space. In the next section we will discuss numerical results with and without the supersymmetry breaking mass (4.4).

\section{Numerical results}

In this section we illustrate the general results of the previous sections for the particular case $N_{f}=2, N_{c}=6$.

In Fig.1 the energy densities vs. redshift are given. We have chosen the same initial condi- 


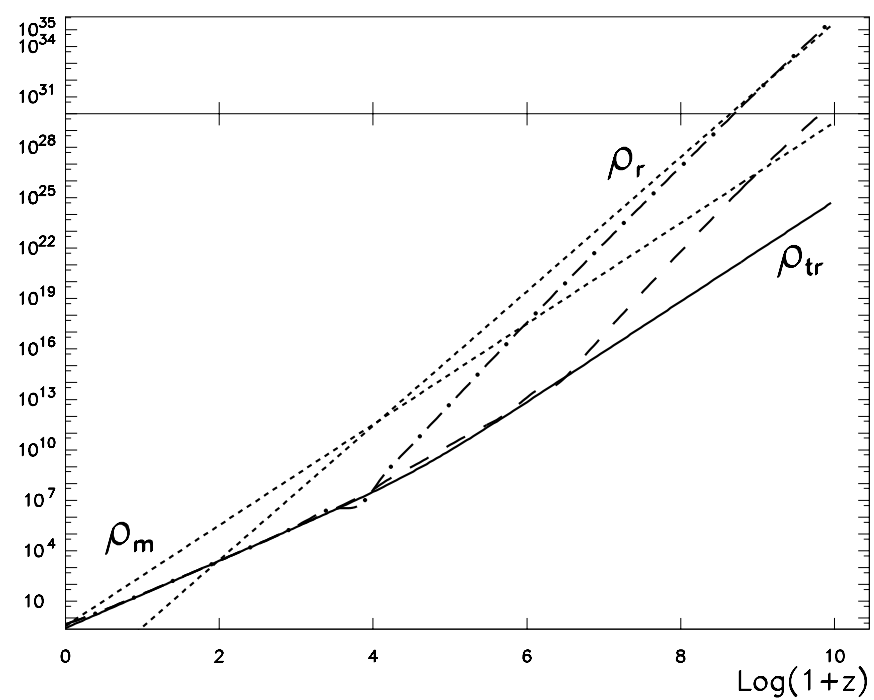

Figure 3: The effect of the interaction with other fields, to be compared with Fig. 1. Adding a term like eq. (4.4) with $\beta_{1}=0.3$ the would-be overshooting solution (dash-dotted line) reaches the tracker in time.

tions for the two VEVs, in order to effectively reproduce the one-scalar case of eq. (2.4), already studied in refs. [10, 11, 16]. No interaction with other fields of the type discussed in the previous section has been considered.

We see that, depending on the initial energy density of the scalar fields, the tracker solution may (long dashed line) or may not (dash-dotted line) be reached before the present epoch. The latter case corresponds to the overshoot solutions discussed in ref. [16], in which the initial scalar field energy is larger than $\rho_{B}$ and the fields are rapidly pushed to very large values. The undershoot region, in which the energy density is always lower than the tracker one, corresponds to $\rho_{c r}^{0} \leq \rho_{Q}^{i n} \leq \rho_{t r}^{i n}$. Thus, all together, there are around 35 orders of magnitude in $\rho_{Q}^{\text {in }}$ at redshift $z+1=10^{10}$ for which the tracker solution is reached before today. Clearly, the more we go backwards in time, the larger is the allowed initial conditions range.

Next, we explore to which extent the twofield system that we are considering behaves as a one scalar model with inverse power-law potential. We have found that, given any initial energy density such that - for $q_{1}^{i n} / q_{2}^{i n}=1$ - the tracker is joined before today, there exists always a limiting value for the fields' difference above which the attractor is not reached in time. In fig. 2 we plot solutions with the same initial energy density but different ratios between the initial values of the two scalar fields.

The effect of the interaction with other fields discussed in Section 4 is shown in Fig.3. Here, we have included the mass term (4.4) during radiation domination with $\beta_{1}=0.3$ and we have followed the same procedure as for Fig.1, searching for undershoot and overshoot solutions. The range of initial energy densities for the solutions reaching the tracker is now enormously enhanced since, as we discussed previously, the fields are now prevented from taking too large values. The same conclusion holds even if different initial conditions for the two fields are allowed, for the same reason.

\section{Acknowledgments}

I thank Antonio Masiero and Massimo Pietroni with whom the results reported in this talk were obtained.

\section{References}

[1] A. Masiero, M. Pietroni and F. Rosati, hep-ph/9905346.

[2] S. Perlmutter et al., astro-ph/9812133; astro-ph/9812473; Nature 391, 51 (1998). See also http://www-supernova.LBL.gov/. 
[3] A.G. Riess et al., astro-ph/9805201.

A.V. Filippenko and A.G. Riess, Phys. Rep. 307 (1998) 31; astro-ph/9905049.

P.M. Garnavich et al, astro-ph/9806396.

B. Leibundgut, G. Contardo, P. Woudt, and J. Spyromilio, astro-ph/9812042.

See also http://cfa-www.harvard.edu/cfa/oir/ Research/supernova/HighZ.html.

[4] J.G. Bartlett, A. Blanchard, M. Le Dour, M. Douspis, and D. Barbosa, astro-ph/9804158.

G. Efstathiou, astro-ph/9904356.

G. Efstathiou, S.L. Bridle, A.N. Lasenby, M.P. Hobson, and R.S. Ellis, astro-ph/9812226.

C.Lineweaver, astro-ph/9805326.

[5] R.G. Carlberg, H.K.C. Yee, and E. Ellingson, Astrophys. J. 478, (1997) 462.

J. Carlstrom, 1999, Physica Scripta, in press.

[6] J.A. Frieman and I. Waga, Phys. Rev. D 57 (1998) 4642.

[7] R.R. Caldwell, R. Dave, and P.J. Steinhardt, Phys. Rev. Lett. 80 (1998) 1582.

[8] M.S. Turner, astro-ph/9904051.

[9] M.S. Turner and M. White, Phys. Rev. D 56 (1997) 4439.

T. Chiba, N. Sugiyama and T. Nakamura, $M N$ $R A S 289$ (1997) L5.

[10] P.J.E. Peebles and B. Ratra, Astrophys. Jour. 325 (1988) L17.

B. Ratra and P.J.E. Peebles, Phys. Rev. D 37 (1988) 3406.

[11] A.R. Liddle and R.J. Scherrer, Phys. Rev. D 59 (1999) 023509.

[12] C. Wetterich, Nucl. Phys. B 302 (1988) 668.

[13] E.J. Copeland, A.R. Liddle, and D. Wands, Phys. Rev. D 57 (1998) 4686.

[14] P.G. Ferreira and M. Joyce, Phys. Rev. Lett. 79 (1997) 4740; Phys. Rev. D 58 (1998) 023503.

[15] I. Zlatev, L. Wang, and P.J. Steinhardt, Phys. Rev. Lett. 82 (1999) 896.

[16] P.J. Steinhardt, L. Wang, and I. Zlatev, Phys. Rev. D 59 (1999) 123504.

[17] C. Baccigalupi and F. Perrotta, Phys. Rev. D 59 (1999) 123508.

W. Hu, D.J. Eisenstein, M. Tegmark, and M. White, Phys. Rev. D 59 (1999) 023512.

A.R.Cooray and D.Huterer, astro-ph/9901097. L. Wang and P.J. Steinhardt, Astrophys. Jour. 508 (1998) 483.

L. Hui, astro-ph/9902275.
B.Ratra, R.Stompor, K.Ganga, G.Rocha, N. Sugiyama, and K.M.Górski, astro-ph/9901014. C. van de Bruck and W. Priester, astro-ph/9810340.

J.S.Alcaniz, J.A.S.Lima, astro-ph/9902298.

[18] L. Wang, R.R. Caldwell, J.P. Ostriker, and P.J.Steinhardt, astro-ph/9901388.

G. Huey, L. Wang, R. Dave, R.R. Caldwell, and P.J. Steinhardt, Phys. Rev. D 59 (1999) 063005. S. Perlmutter, M.S. Turner, and M. White, astro-ph/9901052.

T. Chiba, N. Sugiyama and T. Nakamura, $M N$ $R A S$ vol. 301 (1998) 72.

[19] D.Huterer and M.S.Turner, astro-ph/9808133. T. Nakamura, T. Chiba, MNRAS in press, [astro-ph/9810447].

T. Chiba, T. Nakamura, Prog.Theor.Phys. 100 (1998) 1077.

[20] P. Binétruy, hep-ph/9810553.

[21] J.A. Frieman, C.T. Hill, A. Stebbins, and I. Waga, Phys. Rev. Lett. 75 (1995) 2077.

K. Choi, hep-ph/9902292.

J.E. Kim, hep-ph/9811509.

[22] C. Kolda and D.H. Lyth, hep-ph/9811375.

[23] P. Brax and J. Martin, astro-ph/9905040.

[24] S.M. Carroll, Phys. Rev. Lett. 81 (1998) 3067.

[25] T.R. Taylor, G. Veneziano, and S. Yankielowicz, Nucl. Phys. B 218 (1983) 493.

I. Affleck, M. Dine, and N. Seiberg, Phys. Rev. Lett. 51 (1983) 1026. Nucl. Phys. B 241 (1984) 493.

For a pedagogical introduction see also: M.E.Peskin, hep-th/9702094, TASI 96 lectures.

[26] T. Damour and A.M. Polyakov, Nucl. Phys. B 423 (1994) 532.

[27] T. Damour, gr-qc/9606079, lectures given at Les Houches 1992, SUSY95 and Corfù 1995.

[28] T. Damour and F. Dyson, Nucl. Phys. B 480 (1996) 37.

[29] M. Dine, L. Randall, and S. Thomas, Phys. Rev. Lett. 75 (1995) 398. 